\title{
Non-Markovian Stochastic Epidemics in Extremely Heterogeneous Populations
}

\author{
T. House * \\ Warwick Mathematics Institute, University of Warwick, Coventry, CV4 7AL, UK.
}

\begin{abstract}
A feature often observed in epidemiological networks is significant heterogeneity in degree. A popular modelling approach to this has been to consider large populations with highly heterogeneous discrete contact rates. This paper defines an individual-level non-Markovian stochastic process that converges on standard ODE models of such populations in the appropriate asymptotic limit. A generalised Sellke construction is derived for this model, and this is then used to consider final outcomes in the case where heterogeneity follows a truncated Zipf distribution.
\end{abstract}

Keywords and phrases: Zipf, Sellke, SIR

Mathematics Subject Classification: 92B05, 05C82

\section{Introduction}

A wide variety of different mathematical structures are used to aid modern infectious disease epidemiology, with particular effort expended on capturing the heterogeneities in contact patterns that influence the spread of pathogens $[1,16]$. One of the most active topics in mathematical epidemiology is the use of network theory to represent contact structure $[3,8]$.

In the influential work by Barabási and Albert [4], it was shown that networks generated with preferential attachment to highly connected nodes exhibit power-law behaviour: the probability that a node has $k$ links becomes proportional to $k^{\alpha}$ for large $k$. In the pure Barabási-Albert (BA) model, $\alpha=-3$, although generalisations of this model allow for $-3<\alpha<-2$ [12]. Such distributions have divergent second moment, but finite mean.

Work on synthetic populations [13], sexual behaviour [24] and contact surveys [9] does provide evidence for power-law behaviour; although concerns remain about the statistical problems associated with looking for power laws in empirical data [7].

A crucial quantity in infectious disease epidemiology is the basic reproductive ratio, $R_{0}$, which defines an epidemic threshold such that for $R_{0}>1$ the disease is able to infect an appreciable proportion of a large population [11]. The fact that $R_{0}$ is proportional to the second moment of the contact network degree distribution has been known by mathematical epidemiologists for some time, and is included in the standard textbooks [10]. Just over a decade ago, work by Pastor-Satorras and Vespignani noted that the

${ }^{*}$ Corresponding author. E-mail: T.A.House@warwick.ac.uk

(C) EDP Sciences, 2014 
divergence of the second moment of the degree distribution might lead to divergence of $R_{0}$ and the absence of an epidemic threshold [22], although it was quickly pointed out by May and Lloyd that this need not apply for finite systems [21]. Nevertheless, it is still clear that epidemics on finite networks generated using the BA model have very different behaviour from epidemics in homogeneously mixing populations $[5,23]$, and interest persists in the effects of extreme heterogeneity on epidemic behaviour $[6,17]$.

This paper defines a non-Markovian stochastic process for an epidemic in a finite population with a discrete-valued individual-level measure of heterogeneity in mixing. The Markovian version of this model has as its large-population limit the ODE models used in the 'annealed network' approximation for heterogeneous networks [6]. A generalised Sellke construction is derived for this process to enable fast sampling from the final size distribution. This is used to derive a visualisation of the asymptotic absence of a threshold seen in highly heterogeneous models. Note that the proofs presented are intended to be clear rather than excessively detailed, and so do presuppose some familiarity with standard results in probability and stochastic processes [14].

\section{Methods}

\subsection{A heterogeneous epidemic model}

Consider a population made up of $N$ individuals, indexed by $i, j, \ldots \in \mathbb{N}$. Each individual has an integervalued random variable called the degree, $K_{i} \sim D$, where $D$ is called the degree distribution, representing its level of mixing with the rest of the population. We will write $d_{k}$ for the probability that a random variable with distribution $D$ takes the value $k$. To each individual is assigned a non-negative real-valued infectious period $T_{i} \sim \Gamma$, where $\Gamma$ is called the infectious period distribution. To each individual is assigned a random variable $X_{i}(t)$ representing its disease state, which is either susceptible $S$, infective $I$, or removed $R$. Contacts between individuals $i$ and $j$ happen at the points of a Poisson process with rate $\tau K_{i} K_{j}$. If a susceptible and infective individual contact each other, then the susceptible becomes infective. An individual $i$ that becomes infective at time $t$ is removed at time $t+T_{i}$.

Definition 2.1. A stochastic process satisfying the description above is called a Discrete Heterogeneous Stochastic Epidemic (DHSE).

Note that to specify a DHSE fully requires two distributions, $D$ and $\Gamma$, two parameters, $N$ and $\tau$, and a set of of initial states for the random variables, $\left\{X_{i}(0)\right\}$.

\subsection{Deterministic limit}

\subsubsection{Dynamics}

Consider a continuous-time Markov chain with non-independent integer-valued random variables representing the numbers of the population susceptible and infectious of different degree, $\left\{S_{k}(t), I_{k}(t)\right\}_{k \in \mathcal{K} \subseteq \mathbb{N}}$. There are two types of event and rate:

$$
\begin{aligned}
\left(S_{k}, I_{k}\right) & \rightarrow\left(S_{k}-1, I_{k}+1\right) \text { at rate } \tau k S_{k} \sum_{l \in \mathcal{K}} l I_{l}, \\
I_{k} & \rightarrow I_{k}-1 \text { at rate } \gamma I_{k} .
\end{aligned}
$$

We have $S_{k}(t)+I_{k}(t) \leq N_{k}, \forall t$, and can write $\mathbf{N}=\left(N_{k}\right)$ for the vector of numbers of individuals of degree $k$. At time $t=0$, we pick $\mathbf{N} \sim \operatorname{Multi}(\mathbf{d}, N)$ where $\mathbf{d}=\left(d_{k}\right)$ and 'Multi' is used to stand for the multinomial distribution probability distribution function. This constrains the permissible choice of initial conditions $\left\{S_{k}(0), I_{k}(0)\right\}$.

Definition 2.2. A stochastic process satisfying the description above is called a Discrete Heterogeneous Markovian Epidemic (DHME). 
Note that to specify a DHME fully requires the distribution $D$, two parameters, $N$ and $\tau$, and a set of rules for generation of initial states for the random variables $\left\{S_{k}(0), I_{k}(0)\right\}$ given $\mathbf{N}$.

Theorem 2.3. A DHSE where $\Gamma=\operatorname{Exp}(\gamma)$, i.e. the infectious periods are exponentially distributed with mean $1 / \gamma$, and where $m$ individuals are selected at random to be initial infectives (with other individuals initially susceptible) is equivalent to a DHME with the same D, $N$, and $\tau$.

Proof. Start with a DHSE, and define the lumped variables

$$
S_{k}(t):=\sum_{i} \mathbf{1}_{\left\{X_{i}(t)=S \& K_{i}=k\right\}}, \quad I_{k}(t):=\sum_{i} \mathbf{1}_{\left\{X_{i}(t)=I \& K_{i}=k\right\}} .
$$

The multinomial distribution of $\mathbf{N}$ follows from the definition of the DHSE and the independence of the $\left\{K_{i}\right\}$. Letting $\mathbf{S}=\left(S_{k}\right), \mathbf{I}=\left(I_{k}\right)$, if $m$ individuals are selected at random to be the initial infectives then in the lumped variables this corresponds to a multivariate hypergeometric distribution $\mathbf{I}(0) \sim$

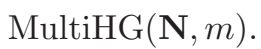

In the DHSE we have the rate of transmission given by a Poisson process, meaning

$$
\operatorname{Pr}\left(X_{i}(t+\delta t)=I \mid X_{i}(t)=S\right)=-\tau K_{i} \sum_{j} K_{j} \mathbf{1}_{\left\{X_{j}(t)=I\right\}} \delta t+o(\delta t)
$$

and (2.1) is simply a lumping of this equation. In the same way, exponential distribution of recovery times is equivalent to a death process on infectives, meaning that

$$
\operatorname{Pr}\left(X_{i}(t+\delta t)=R \mid X_{i}(t)=I\right)=\gamma \delta t+o(\delta t),
$$

and (2.2) is a lumping of this process.

Theorem 2.4. A DHME with finite $\mathcal{K}$ and $D$ independent of $N$ has its deterministic large $N$ limit given by an $O D E$ system of the form

$$
\frac{\mathrm{d} s_{k}}{\mathrm{~d} t}=-\beta k s_{k} \sum_{l} l_{\iota_{l}}, \quad \frac{\mathrm{d} \iota_{k}}{\mathrm{~d} t}=\beta k s_{k} \sum_{l} l_{\iota_{l}}-\gamma \iota_{k} .
$$

Proof. Note that the rates (2.1) and (2.2) can be written in the form

$$
\begin{aligned}
\left(S_{k}, I_{k}\right) & \rightarrow\left(S_{k}-1, I_{k}+1\right) \text { at rate }(\tau N) N k\left[\frac{S_{k}}{N}\right] \sum_{l \in \mathcal{K}} l\left[\frac{I_{l}}{N}\right], \\
I_{k} & \rightarrow I_{k}-1 \text { at rate } \gamma N\left[\frac{I_{k}}{N}\right] .
\end{aligned}
$$

Defining

$$
s_{k}(t):=\mathbb{E}\left[\frac{S_{k}}{N}\right], \quad \iota_{k}(t):=\mathbb{E}\left[\frac{I_{k}}{N}\right], \quad \beta:=\tau N
$$

we can then apply the results of Kurtz $[18,19]$ to derive the deterministic limit (2.6) with corrections appearing at $O\left(N^{-1 / 2}\right)$. 


\subsubsection{Early behaviour}

Analysis of (2.6) using dynamical systems theory shows that perturbations away from the disease-free equilibrium $(\mathbf{s}, \boldsymbol{\iota})=(\mathbf{d}, \mathbf{0})$, grow exponentially with rate

$$
r=\beta \mathbb{E}_{D}\left[K^{2}\right]-\gamma,
$$

provided $r$ is positive. The argument made by Pastor-Satorras and Vespignani [22] is then essentially that if the second moment of $D$ is divergent, then (2.10) implies that an arbitrarily small $\beta$ can still lead to a growing epidemic.

Note that equations like (2.6) have been used for some time in epidemiological modelling [20]. In a more general sense this system represents a special, discrete version of the proportionate mixing analysed in the non-Markovian case by [10] and so using this analysis the quantity

$$
\rho:=\beta \mathbb{E}_{D}\left[K^{2}\right] \mathbb{E}_{\Gamma}[T]
$$

will be an appropriate definition for the basic reproductive ratio $R_{0}$ of the large- $N$ DHSE: we expect a large epidemic exactly when $\rho>1$. We will make use of the definition (2.11) in the finite case where this is not a 'threshold' but remains an appropriate quantity to use when comparing different parameter values.

\subsubsection{Late behaviour}

Following the broad approach of [17, Appendix B], it is possible to manipulate (2.6) in the limit where the initial level of infection is very small and obtain the expression

$$
s_{k}^{\infty}:=\lim _{t \rightarrow \infty} s_{k}(t)=d_{k}\left(\exp \left(-\frac{\beta}{\gamma} \sum_{l} l\left(d_{l}-s_{l}^{\infty}\right)\right)\right)^{k} .
$$

Then the expected final proportion of individuals in the susceptible class at the end of the epidemic is

$$
s^{\infty}=G(\psi), \quad \text { for } \quad \psi=\exp \left(-\frac{\beta}{\gamma}\left(\mathbb{E}_{D}[K]-\psi G^{\prime}(\psi)\right)\right),
$$

where $G$ is the probability generating function of $D$. Looking at this transcendental equation, solutions for which $\psi<1$ will only exist if $r>0$ for $r$ as in (2.10). Further consideration of these equations leads us to expect a change in behaviour around $\rho=1$, if such a change exists, to be percolation-like.

\subsection{A generalised Sellke construction}

Consider a population made up of $N$ individuals, indexed by $i, j, \ldots \in \mathbb{N}$. Each individual has an integer-valued random variable $K_{i} \sim D$. Each individual also picks a non-negative real-valued infectious period $T_{i} \sim \Gamma$, and has a random variable $X_{i}(t) \in\{S, I, R\}$ representing its disease state. Each initially susceptible individual picks a resistance $Q_{i} \sim \operatorname{Exp}\left(K_{i}\right)$, with initial infectives having zero resistance. The infectious pressure of the epidemic at time $t$ is defined as

$$
\Lambda(t):=\tau \int_{0}^{t} \sum_{j=1}^{N} K_{j} \mathbf{1}_{\left\{X_{j}(u)=I\right\}} d u .
$$

An initially susceptible individual becomes infectious at the first time $t$ when $Q_{i}<\Lambda(t)$.

Definition 2.5. A stochastic process satisfying the description above is called a Discrete Heterogeneous Sellke Construction (DHSC).

Note that to specify a DHSC fully requires the same quantities as the DHSE: two distributions, $D$ and $\Gamma$, and two parameters, $N$ and $\tau$, and a set of initial states for the random variables, $\left\{X_{i}(0)\right\}$. 
Lemma 2.6. A DHSE and DHSC with the same $D, \Gamma, N, \tau$ and $\left\{X_{i}(0)\right\}$ are equivalent.

Proof. From the conditions stated, the only difference between the DHSE and DHSC is in the behaviour of transmission. We can follow the broad exposition in [2]. First, note that in the DHSC

$$
Q_{i} \sim \operatorname{Exp}\left(K_{i}\right) \quad \Rightarrow \quad \operatorname{Pr}\left(Q_{i}>q\right)=\mathrm{e}^{-K_{i} q} .
$$

Then over a time period $[t, t+\delta t]$ we know that

$$
\begin{aligned}
\operatorname{Pr}\left(Q_{i}>\Lambda(t+\delta t) \mid Q_{i}>\Lambda(t)\right) & =\exp \left(-(\Lambda(t+\delta t)-\Lambda(t)) K_{i}\right) \\
& =1-\tau K_{i} \sum_{j} K_{j} \mathbf{1}_{\left\{X_{j}(t)=I\right\}}+o(\delta t) .
\end{aligned}
$$

In the DHSE, we know from the behaviour of Poisson processes that

$$
\operatorname{Pr}\left(X_{i}(t+\delta t)=S \mid X_{i}(t)=S\right)=1-\tau K_{i} \sum_{j} K_{j} \mathbf{1}_{\left\{X_{j}(t)=I\right\}}+o(\delta t)
$$

Therefore the behaviour of transmission in the two models is equivalent.

Theorem 2.7. If $Z$ is the final number of removed individuals in a DHSE

$$
Z:=\lim _{t \rightarrow \infty} \sum_{j=1}^{N} \mathbf{1}_{\left\{X_{j}(t)=R\right\}}
$$

then by picking degrees, resistances and recovery times as in the DHSC, and defining the indexing such that $Q_{i} \leq Q_{i+1}, \forall i$,

$$
Z=\min \left\{i \mid Q_{i+1}>\tau \sum_{j=1}^{i} K_{j} T_{j}\right\} .
$$

Proof. From Lemma 2.6, the DHSE is equivalent to the DHSC. Permutation of the indices can be performed without loss of generality since there is no dependence of $D$ or $\Gamma$ on $i$. Consideration of the integral (2.14) gives

$$
\int_{0}^{\infty} \mathbf{1}_{\left\{X_{i}(u)=I\right\}} d u= \begin{cases}T_{i} & \text { if } i \text { is ever infected } \\ 0 & \text { if } i \text { is never infected }\end{cases}
$$

Then from the choice of indexing,

$$
Q_{i+1}>\tau \sum_{j=1}^{i} K_{j} T_{j}=: \Lambda_{\infty} \quad \Rightarrow \quad Q_{J^{\prime}>i}>\Lambda_{\infty}=\lim _{t \rightarrow \infty} \Lambda(t) .
$$

This means that the first individual to resist infection will determine the final size as in (2.19).

\section{Results and Discussion}

The major practical benefit to Theorem 2.7 is the enhanced numerical performance of the Sellke construction compared to direct simulation [15]. We use this to simulate the final size of a DHSE where $D$ is a truncated Zipf distribution, i.e.

$$
d_{k}=\kappa_{\alpha} k^{\alpha}, \quad \text { for } \quad \kappa=\sum_{k=1}^{k_{\max }} k^{\alpha} .
$$



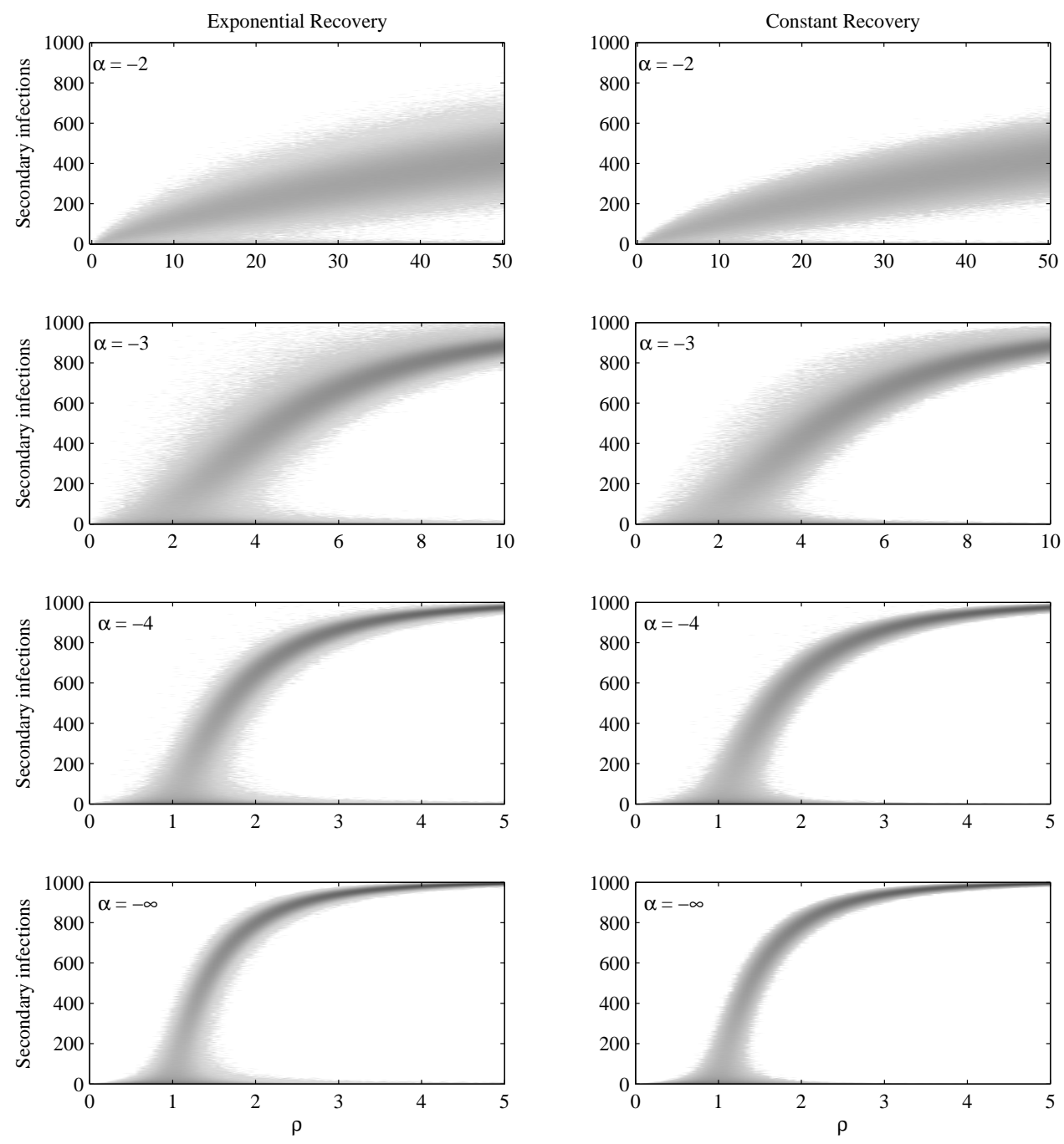

FiguRE 1. Numerical results for the final size distribution for exponential mean 1 and constant unit infectious period, truncated Zipf degree distribution, a total population size $N=10^{3}, m=1$ initial infective, and $10^{5}$ realisations for each value of $\alpha, \rho$, and distribution. Colour intensity is proportional to probability ${ }^{1 / 6}$

In the limit $\alpha \rightarrow-\infty$, this reduces the heterogeneous model to the standard 'mass action' epidemic where each individual has $K_{i}=1$. But for smaller $|\alpha|$, the $\lceil 1-\alpha\rceil$-th moment will grow with $k_{\max }$ rather than converging. For $-3<\alpha \leq-2$, i.e. a second moment that diverges for large $k_{\max }$, this would therefore be expected not to exhibit threshold behaviour.

The DHSC can be used to sample efficiently from the final size distribution of the epidemic. Figure 1 shows the results of this simulation, for constant and exponential $\Gamma$ as well as $\alpha=-2,-3,-4,-\infty$, when the natural choice $k_{\max }=N-1$ is made. This visualises how low values of $|\alpha|$ are associated with 
less critical-looking behaviour at $\rho=1$. Note that this is not in contradiction with Theorem 2.4, and the analysis of the deterministic limit threshold, which requires finite $\mathcal{K}$. In the current context this means that $k_{\max } \ll N$, which does not hold in the simulations and highlights the problems of reliance on ODE methods alone.

In conclusion, it is possible to provide a mathematically transparent model of extreme discrete heterogeneity, which allows fast sampling from the final size distribution to allow insights into the subtle epidemiological phenomena associated with extreme heterogeneity in mixing.

Acknowledgements. Work funded by the UK Engineering and Physical Sciences Research Council. I would like to thank the anonymous reviewer for helpful comments.

\section{References}

[1] R. M. Anderson, R. M. May. Infectious Diseases of Humans. Oxford University Press, Oxford, 1991.

[2] H. Andersson, T. Britton. Stochastic Epidemic Models and Their Statistical Analysis, volume 151 of Springer Lectures Notes in Statistics. Springer, Berlin, 2000.

[3] S. Bansal, B. T. Grenfell, L. A. Meyers. When individual behaviour matters: homogeneous and network models in epidemiology. Journal of the Royal Society Interface, 4 (2007), no. 16, 879-91.

[4] A. L. Barabási, R. Albert. Emergence of scaling in random networks. Science, 286 (1999), no. 5439, 509-512.

[5] N. Berger, C. Borgs, J. T. Chayes, A. Saberi. On the spread of viruses on the internet. In Proceedings of the 16th Symposium on Discrete Algorithms, 2005.

[6] C. Castellano,R. Pastor-Satorras. Thresholds for epidemic spreading in networks. Physical Review Letters, 105 (2010), no. $21,218701$.

[7] A. Clauset, C. R. Shalizi, M. E. J. Newman. Power-law distributions in empirical data. SIAM Review, 51 (2009), no. 4, 661-703.

[8] L. Danon, A. P. Ford, T. House, C. P. Jewell, M. J. Keeling, G. O. Roberts, J. V. Ross, and M. C. Vernon. Networks and the epidemiology of infectious disease. Interdisciplinary Perspectives on Infectious Diseases, (2011), 1-28.

[9] L. Danon, T. House, M. J. Keeling, J. M. Read. Social encounter networks: collective properties and disease transmission. Journal of the Royal Society Interface, 9 (2012), no. 76, 2826-2833.

[10] O. Diekmann, J. A. P. Heesterbeek. Mathematical epidemiology of infectious diseases: Model building, analysis and interpretation. John Wiley \& Sons Ltd., 2000.

[11] O. Diekmann, J. A. P. Heesterbeek, J. A. J. Metz. On the definition and the computation of the basic reproduction ratio $R_{0}$ in models for infectious diseases in heterogeneous populations. Journal of Mathematical Biology, 28 (1990), no. $4,365-382$.

[12] R. Durrett. Random Graph Dynamics. Cambridge University Press, 2007.

[13] S. Eubank, H. Guclu, V. S. A. Kumar, M. V. Marathe, A. Srinivasan, Z. Toroczkai, N. Wang. Modelling disease outbreaks in realistic urban social networks. Nature, 429 (2004), no. 6988, 180-184.

[14] G. R. Grimmett, D. R. Stirzaker. Probability and Random Processes. Oxford University Press, 3rd edition, 2001.

[15] T. House, J. V. Ross, D. Sirl. How big is an outbreak likely to be? Methods for epidemic final-size calculation. Proceedings of the Royal Society A, 469 (2013), no. 2150, 20120436.

[16] M. J. Keeling, P. Rohani. Modeling Infectious Diseases in Humans and Animals. Princeton University Press, New Jersey, 2007.

[17] I. Z. Kiss, D. M. Green, R. R. Kao. The effect of contact heterogeneity and multiple routes of transmission on final epidemic size. Mathematical Biosciences, 203 (2006), no. 1, 124-36.

[18] T. G. Kurtz. Solutions of ordinary differential equations as limits of pure jump Markov processes. Journal of Applied Probability, 7 (1970), no. 1, 49-58.

[19] T. G. Kurtz. Limit theorems for sequences of jump Markov processes approximating ordinary differential processes. Journal of Applied Probability, 8 (1971), no. 2, 344-356.

[20] R. M. May, R. M. Anderson. The transmission dynamics of human immunodeficiency virus (HIV). Philosophical Transactions of the Royal Society of London Series B, 321 (1988), no. 1207, 565-607.

[21] R. M. May, A. L. Lloyd. Infection dynamics on scale-free networks. Physical Review E, 64 (2001), 066112.

[22] R. Pastor-Satorras, A. Vespignani. Epidemic dynamics and endemic states in complex networks. Physical Review E, 63 (2001), 066117.

[23] R. Pastor-Satorras, A. Vespignani. Epidemic dynamics in finite size scale-free networks. Physical Review E, 65 (2002), no. 3, 035108.

[24] A. Schneeberger, C. H. Mercer, S. A. J. Gregson, N. M. Ferguson, C. A. Nyamukapa, R. M. Anderson, A. M. Johnson, G. P. Garnett. Scale-free networks and sexually transmitted diseases: a description of observed patterns of sexual contacts in Britain and Zimbabwe. Sexually Transmitted Diseases, 31 (2004), no. 6, 380-7. 


\section{A. Code for the DH Sellke construction}

The following MATLAB function accepts a vector of degree numbers K, a number of initial infectives $\mathrm{m}$, a transmission rate tau, and a recovery rate gamma. If the final parameter is set to NaN, a fixed unit recovery time is used. A sample from the final size distribution is returned.

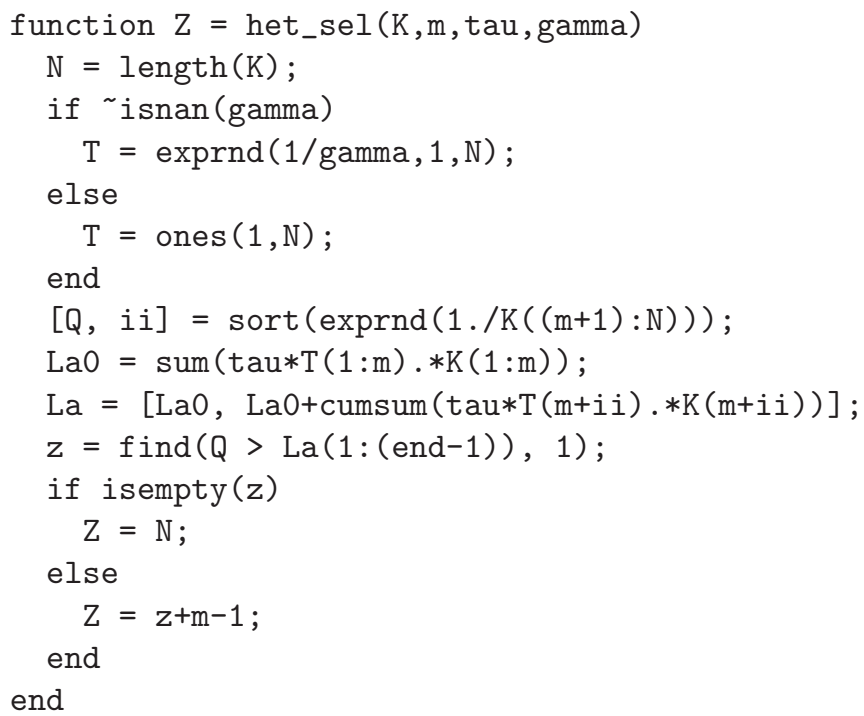

